\title{
Une vie de palais: la cour du cardinal Alexandre Farnèse vers 1563
}

\author{
PIERRE HURTUBISE
}

L e document qui est à l'origine de la présente étude est un budget dressé par les services d'intendance du cardinal Alexandre Farnèse pour l'année 1563. ${ }^{1} \mathrm{Ce}$ document m'avait servi il y a environ une dizaine d'années à préparer un article sur un collègue et contemporain d'Alexandre Farnèse, le cardinal Bernardo Salviati, article paru dans les Mélanges de l'École Française de Rome en 1980 sous le titre: "La table d'un cardinal de la Renaissance: Aspects de la cuisine et de l'hospitalité à Rome au milieu du XVI ${ }^{\mathrm{e}}$ siècle."' Mais, à l'époque, je n'avais fait de ce document qu'un usage très limité, me contenant d'un certain nombre d'éléments susceptibles d'éclairer mon propos qui était d'illustrer ce que pouvait être le train de vie d'une maison cardinalice à Rome au $\mathrm{XVI}^{\mathrm{e}}$ siècle, mais d'une maison se situant dans la bonne moyenne. Comme je l'écrivais à l'époque, "Bernardo Salviati n'est ni un Alexandre Farnèse, ni un Hippolyte ou un Louis d'Este $[\ldots]$ ces incomparables seigneurs de la Rome de la deuxième moitié du XVI ${ }^{\mathrm{e}}$ siècle," mais ce que nous savons du train de vie de sa maison correspond sans doute "mieux à la réalité de ce qui se passait au jour le jour" dans la majorité des cours cardinalices de la Rome de l'époque que chez ces quelques fastueux personnages. ${ }^{3}$

Mais si fastueux et apparemment si exceptionnels ces derniers puissent-ils nous paraître, ils n'en méritent pas moins eux aussi de retenir notre attention, ne fût-ce que pour satisfaire notre curiosité, curiosité d'autant plus légitime d'ailleurs que le faste, voir les excès qu'on leur prête habituellement sont le plus souvent fondés sur des impressions ou encore des instantanés d'époque qui sont loin de représenter toute la réalité, en particulier la réalité quotidienne. Mais peut-être ces personnages méritent-ils surtout d'être connus en raison du rôle "emblématique" qu'ils ont joué en leur temps: c'est sur eux qu'on se modelait et, en définitive, par rapport à eux qu'on était jaugé, même si, en pratique, faute de moyens, on était très souvent obligé de se contenter de pâles ou modestes imitations. Leur performances, par rapport à celles de 
la grande majorité de leurs collègues, peuvent paraître exceptionnelles et, de fait, par bien des côtés, l'étaient, mais ils n'en constituaient pas pour autant eux-mêmes des exceptions à la règle, car, pour les contemporains, ils étaient ou, mieux, ils incarnaient en quelque sorte la règle, et c'est cette règle qui, de fait, souffrait de nombreuses exceptions en la personne de collègues moins bien nantis ou encore moins libéraux qu'eux.

De ce point de vue, il n'est pas sans intérêt de noter que notre document de départ, c'est-à-dire le budget d'Alexandre Farnèse pour 1563, provient des papiers du cardinal Bernardo Salviati et qu'il entra en possession de ce dernier au moment où il était lui-même à organiser sa cour à Rome suite à sa promotion au cardinalat en $1561 .{ }^{4}$ Il est permis de penser que le document en question servit à cette organisation, même si le modèle qui y est proposé était destiné à n'être et ne sera de fait, pour des raisons que j'ai expliquées ailleurs, ${ }^{5}$ que très imparfaitement imité.

$\mathrm{Si}$ quelques grands cardinaux ont joué au $\mathrm{XVI}^{\mathrm{e}}$ siècle le rôle emblématique que je viens de décrire, nul ne l'a fait plus volontiers, avec plus de panache et surtout de façon plus soutenue que le cardinal Alexandre Farnèse. D'où l'intérêt que présente le budget de 1563 pour l'étude de la vie de cour à Rome au milieu du $\mathrm{XVI}^{\mathrm{e}}$ siècle.

\section{Quelques données essentielles}

Petit-fils du pape Paul III (1534-1549), Alexandre Farnèse sera fait cardinal dès l'élection de ce dernier au trône pontifical à l'automne de 1534 , alors qu'il n'avait que 14 ans. Népotisme oblige. Une année plus tard, il obtiendra le poste de vice-chancelier de l'Église jusque-là détenu par le cardinal Ippolito de' Medici, poste de très grand prestige, mais également fort rémunérateur qu'il conservera jusqu'à sa mort en 1589 et qui fera de lui un des personnages les plus importants et les plus influents de l'Église. D'autres dignités, d'autres bénéfices viendront s'ajouter par la suite, lui octroyant par le fait même un supplément de prestige et de pouvoir qui lui permettront de fait de jouer un rôle de tout premier plan par rapport à la politique pontificale, au moment des conclaves en particulier, mais également sous les règnes de certains des successeurs de son aïeul, Paul III. Il est, sans conteste, une des figures les plus en vue, sinon la plus en vue de Rome entre 1535 et $1589 .^{6}$

Au poste de vice-chancelier était rattachée une des plus belles résidences de Rome, soit l'ancien palais Riario devenu depuis 1517 palais de la Chancellerie. ${ }^{7}$ C'est dond là que viendra s'installer le jeune Alexandre Farnèse en 1535, d'autant plus que le palais Farnèse où il avait habité jusque-là, sous 
l'oeil vigilant de son aïeul le pape, était redevenu cette même année un immense chantier, suite à la décision de Paul III de donner à ce monument familial des dimensions et une apparence dignes du rang que les Farnèse, grâce à lui, occupaient désormais à Rome. ${ }^{8}$ Alexandre Farnèse retournera éventuellement habiter le palais familial, mais une trentaine d'années plus tard seulement, une fois complétés les principaux travaux de réaménagement entrepris à la demande de son aïeul. Entre-temps, le palais Farnèse sera à la disposition du reste de la famille, en particulier de son frère cadet, Ranuccio (1530-1565), fait cardinal en $1545 .^{9}$

Le budget de 1563 nous permet de connaître avec passablement d'exactitude le train de vie d'Alexandre Farnèse, de même que celui de l'imposante famille qu'il entretenait à ses frais au palais de la Chancellerie. Cette famille était composée à l'époque d'environ 270 personnes dont une trentaine de gentilshommes et de prélats, une soixantaine d'officiers au moins et quelque 170 serviteurs. ${ }^{10}$ Sans doute une des cours les plus imposantes à Rome, à l'époque, après celle du pape. ${ }^{11}$

\section{A. Budget du cardinal Farnèse (en écus)}

\begin{tabular}{|c|c|c|c|}
\hline Entrées & Brutes & Nettes & $\%$ \\
\hline Italie & 56733 & 54133 & $(74,44)$ \\
\hline France & 22248 & 16677 & $(23,56)$ \\
\hline TOTAL & 78981 & 70820 & $(100)$ \\
\hline \multicolumn{4}{|l|}{ Sorties } \\
\hline \multicolumn{4}{|c|}{$\begin{array}{l}\text { Charges diverses (pensions, } \\
\text { cens, intérêts sur emprunts }\end{array}$} \\
\hline & & 8666 & $(19,03)$ \\
\hline Dépenses & elles & 4300 & $(9,44)$ \\
\hline Dépenses & lières & 4230 & $(9,29)$ \\
\hline \multicolumn{4}{|c|}{ Intendance du palais et } \\
\hline TOTAL & & 45539 & $(100)$ \\
\hline
\end{tabular}


Comme on peut le voir par le tableau ci-dessus (A), Alexandre Farnèse disposait à l'époque d'un revenu brut de près de 80000 écus par an. ${ }^{12}$ Vingt ans plut tôt, ce revenu était d'environ 60000 écus, ${ }^{13}$ vingt ans plus tard, il sera de près de 120000 écus. ${ }^{14} \mathrm{Bel}$ exemple d'un prélat qui savait tirer avantage du rang qu'il occupait et de l'influence qu'il avait dans l'Église et la société du temps. De ce total de 80000 écus, il lui fallait déduire un certain nombre de charges liées à l'administration des bénefices dont il disposait en France et en Italie, soit, en 1563, plus de 8000 écus par an, de même que près de 1900 écus de pensions et environ 6800 écus d'intérêts sur emprunts ou hypothèques. Il pouvait donc compter sur quelque 62000 écus nets par an pour répondre à ses propres besoins de même qu'aux besoins de sa cour. ${ }^{15}$ Quels étaient ces besoins? Commençons par ce qui le touchait ou concernait de plus près. Le vêtement, tout d'abord. Le cardinal y consacrait près de 900 écus par an, dont 360 pour sa propre garde-robe, le reste pour celle de ses pages, valets de chambre et palefreniers. Plus de 1000 écus partaient en aumônes; quelque 300 servaient à financer les déplacements habituels du prélat: sans doute ses nombreuses "escapades" dans le Latium ou dans les environs immédiats de Rome. Mais il y avait aussi 1200 écus prévus pour ses dépenses personnelles en plus de 1440 écus de dépenses dites extraordinaires dont la plus grande partie était nul doute destinée aux "menus plaisirs" dont le cardinal tenait à agrémenter ses journées de même que celles de ses hôtes ou encore aux "largesses" qu'il jugeait utiles, voire indispensables de se permettre. Tels ces divertissements offerts à l'occasion dans son jardin du Transtevère ${ }^{16}$ ou, mieux, à Caprarola $;{ }^{17}$ tels ces somptueux cadeaux présentés au moment opportun à des proches ou encore à des grands ou influents personnages; ${ }^{18}$ tels ces beaux objets ou ces agréables passe-temps qu'il aimait de temps à autre s'offrir à lui-même. ${ }^{19}$

Mais il lui fallait aussi assumer les coûts d'aménagement et d'entretien des immeubles et autres propriétés qui lui servaient tout à la fois de lieux de résidence, de travail et de loisirs. Le seul coût des réparations à faire chaque année au palais de la Chancellerie s'élevait à 300 écus; les vignes de Pratti, Torretta et Villanova, situées dans la proche banlieue romaine, de même que le jardin du Transtevère, plus haut mentionné, représentaient des coûts d'entretien de plus de 330 écus; quant à l'incomparable villa de Caprarola, alors en construction près de Viterbe sous la direction du célèbre Vignola, elle dévorait, à elle seule et au seul titre des frais de construction, quelque 3600 écus par an.

$\mathrm{Si}$, délaissant un moment la personne du cardinal et ses obligations comme gestionnaire du capital immobilier plus haut décrit, nous nous intéressons 
maintenant à l'intendance comme telle du palais de la Chancellerie et de la nombreuse "famille" qui y réside, nous découvrons que ce poste représente et de loin l'item principal du budget d'Alexandre Farnèse, soit 28343 écus au total ou $62 \%$ de toutes les dépenses inscrites à ce budget.

\section{Coût d'intendance du palais et de la "famille" (en écus)}

$\begin{array}{lrr}\text { Salaires et 'provisions' } & 4585 & (16,18 \%) \\ \text { Fournitures } & 2484 & (8,76 \%) \\ \text { Écurie } & 3474 & (12,26 \%) \\ \text { Cuisine } & 17800 & (62,80 \%) \\ & & \\ \text { TOTAL } & 28343 & (100 \%)\end{array}$

Les salaires des officiers et simples serviteurs de mêmes que les "provisions" de prélats, gentilshommes et autres proches collaborateurs du cardinal entraînent un déboursé annuel de 4585 écus. ${ }^{20}$ Les diverse fournitures de maison, y compris la chandelle (192 écus), le bois (780 écus), les médicaments (360 écus) auxquels, pour faire complet, il faudrait sans doute ajouter les vêtements des pages, valets de chambre et palefreniers mentionnés plus haut (504 écus) occasionnent chaque année des dépenses de 2484 écus. Le service de l'écurie coûte pour sa part 3474 écus l'an, cela comprenant la nourriture des 109 montures (97 chevaux, 9 mules et trois ânes) à la disposition du cardinal et de sa famille (2 754 écus), diverses fournitures (504 écus) et l'entretien des coches (86 écus), ces nouveaux modes de locomotion désormais présents dans toutes les grandes maisons de Rome. ${ }^{21}$

\section{Plaisirs et prestige de la table}

Mais le poste de loin de plus important du budget d'Alexandre Farnèse, c'est, comme dans la plupart des cours de l'époque, la cuisine. En effet, près de 18000 écus sont consacrés chaque année à ce seul item, soit $39 \%$ du budget total et $63 \%$ de la partie consacrée à l'intendance comme telle du palais. Nous avons la chance de connaître ce poste de façon extrêmement précise et détaillée. Non seulement nous fournit-on la liste complète des aliments consommés chaque jour au palais de la Chancellerie, mais on va jusqu'à fournir en plus le détail des menus servis midi et soir à la table du cardinal. Aussi avons-nous estimé important de nous attarder quelque peu sur cet aspect bien particulier de l'intendance de l'ancien palais Riario. 
Quelques données quantitatives, tout d'abord. Côté vin, il se consomme chaque année chez le cardinal Farnèse 3420 barils ou plus de 2000 hectolitres de ce précieux liquide représentant une consommation quotidienne brute de 1,62 litre par personne. On y mange chaque douze mois 516 rubbia de pain, soit environ $112000 \mathrm{kgs}$, ce qui équivaut à une consommation de 1136 grammes par tête par jour. Et cela sans compter les 84 rubbia ou $18228 \mathrm{kgs}$ destinés chaque année à la meute de chien du cardinal. Quant à la consommation d'huile, elle s'établit à environ 4200 litres par an, soit, une base quotidienne, 4,26 cl par personne. ${ }^{22}$ Enfin, 165992 livres, ou $56269 \mathrm{kgs}$ de viande sont servis chaque année aux habitants et hôtes du palais pour une consommation brute par personne d'environ 2,62 livres ou 887 grammes. $^{23}$

Nous sommes moins bien informés relativement à la consommation de poisson car notre document se limite à nous dire ce qu'il en était durant la carême seulement et pour la seule table du cardinal, de sorte qu'il est presque impossible de déterminer la quantité de poisson consommée par l'ensemble de la famille. A la table du cardinal, sans doute mieux pourvue que celle des simples serviteurs, il semble que la consommation quotidienne par personne ait été de 0,62 livres ou 211 grammes environ. ${ }^{24} \mathrm{Ce}$ qui paraît assez peu en regard de la consommation de viande plus haut indiquée, mais n'oublions pas que les jours maigres, durant le carême du moins, étaient aussi, dans les régions catholiques de l'époque, des jours de jeûne, donc de consommation passablement réduite.

Si nous comparons ses données à celles de la cour de Bernardo Salviati, plus haut mentionné, de notables différences sautent immédiatement aux yeux. Sans doute la consommation d'huile est-elle légèrement inférieure chez le cardinal Farnèse, mais celle de vin, puis celle de pain et de viande - ces éléments-clés de la "bonne" cuisine d'époque - sont par contraste, beaucoup plus importants chez lui que chez le cardinal Salviati, comme en témoigne le tableau ci-dessous.

C. Consommation quotidienne brute par personne $e^{25}$

\begin{tabular}{lcrr}
\hline Denrée & $\begin{array}{c}\text { Palais de la } \\
\text { Chancellerie }\end{array}$ & $\begin{array}{c}\text { Palais } \\
\text { Salviati }\end{array}$ & Valladolid \\
\hline Pain & $1136 \mathrm{gr}$. & $900 \mathrm{gr}$. & $443 \mathrm{gr}$. \\
Vin & $1,62 \mathrm{l}$ & $0,85 \mathrm{l}$ & $0,25 \mathrm{l}$ \\
Viande & $887 \mathrm{gr}$. & $573 \mathrm{gr}$. & $75 \mathrm{gr}$. \\
Huile & $4,26 \mathrm{cl}$ & $6 \mathrm{cl}$ & $0,54 \mathrm{cl}$
\end{tabular}


Et si nous pouvions, comme nous avons pu le faire pur la cour de Bernardo Salviati, distinguer la consommation par catégories de personnes, combien plus accentué nous paraîtrait le contraste entre l'un et l'autre de ces régimes alimentaires. Malheureusement la documentation dont nous disposons ne permet pas, si ce n'est dans quelques rares cas, d'aller jusque-là. Cela dit, les marges révélées par notre tableau sont trop importantes pour ne pas être significatives.

Mais ces marges ne disent pas tout. En effet, il faut aussi se poser le problème de savoir de quelle qualité étaient les aliments servis à la table du cardinal Farnèse par rapport à ce que l'on trouvait ailleurs à la même époque. Et ici, nous sommes merveilleusement bien renseignés, beaucoup mieux en tout cas que pour d'autres cours, y compris celle du cardinal Salviati, puisque, comme nous l'avons indiqué plus haut, nou possédons le détail des menus servis midi et soir chez Alexandre Farnèse.

Sa table rassemblait chaque jour environ 90 convives. Sas doute faut-il inclure dans ce groupe sélect les prélats, gentilshommes et principaux officiers de la maison de même que les invités de marque dont le cardinal tenait sans doute assez souvent à s'entourer. ${ }^{26}$ Alors que les autres membres de la famille devaient se contenter d'une pitance relativement modeste comprenant tout de même pain, vin, viande, potage, huile et épices en plus d'un companatico en argent de 4,36 baiochhi par personne par jour, les commensaux du cardinal avaient droit à un riche éventail de mets diversement, voir, dans certains cas, somptueusement apprêtés, comprenant habituellement, en plus du vin servi, nous l'avons vu, en abondance, potages, pâtes, légume, salades, fromages, fruits et amandes, pâtisseries, puis surtout, en carême, poissons et fruits de mer, hors carême, viandes et abâts de toutes sortes. ${ }^{27}$

Veau et mouton sont particulièrement à l'honneur les jours où on fait gras, mais les menus comprennent toujours en plus, au choix des convives, chevreau, chapon, poulet, pigeon, oison, dindonneau de même que certaines spécialités charcutières, telles ris de veau, testicules d'agneau, pieds de veau, pâtés et saucissons. Les jours maigres, nécessairement plus frugaux, en période de carême surtout, en raison de l'obligation concomitante de jeûner, ne sont pas pour autant des jours de moindre raffinement. Qu'on en juge.

Tout d'abord, le midi, on se voit servir, comme plat principal, ou de la spigola, ${ }^{28}$ ou de l'ombrine, ou du corbeau de mer ou quelque autre poisson rare bouilli, également du rotangle, de la daurade et du rouget frits, du mulet ou du brochet cuit sur le gril, pris, à titre d'entrée sans doute, du hareng blanc ou du brochet fumé, du crabe, des tellines, en plus d'une soupe au poisson de rivière, d'une soupe aux jeunes calmars et d'un potage aux légumes. Il est 
également fait mention de plongeons ("marangole", ${ }^{29}$ ) qui, à titre d'oiseaux aquatiques, avaient droit de figurer aux menus de carême, mais nous ne saurions dire si ces volatiles étaient servis comme entrée ou comme plat principal ni de quelle façon ils étaient apprêtés. Chose certaine, leur présence devrait ajouter sinon à la qualité, du moins à l'originalité du menu en question.

Le soir, on doit se contenter, comme plat principal, de hareng, de quelques tranches de thon ou de saumon et d'une sorte de poisson de rivière, mais on se voit également offrir, comme antipasti sans doute, tellines ou escargots, crabe ou homard, anchois ou anguilles, en plus d'un potage blanc au houblon et d'un potage aux épinards et au fenouil.

Le cardinal, pour sa part, a droit à quelques plats préparés spécialement pour lui au repas du midi, un plat de poisson de rivière et un autre de jeunes calmars, au repas du soir, un plat de tortue, et de nouveau, un plat de jeunes calmars - manifestement Alexandre Farnèse avait un faible pour ce mollusque - auxquels venait peut-être s'ajouter ce "plat délicat" non spécifié, indiqué comme faisant chaque jour partie du menu vespéral.

Côté fruits, on semble s'être contenté le midi de brugons et de raisins secs; le soir, au contraire, la table se couvre de poires, de pommes roses et de pommes d'api, de raisin de Damas, de figues, de marrons et de cardons, en plus d'amandes, de pistaches et de pignons. Même s'ils ne sont pas mentionnés comme tels dans les menus hors carême, hormis les amandes et les raisins secs, la plupart de ces fruits et d'autres sans doute provenant des vergers du cardinal devaient y trouver place au gré des diverses saisons.

Quant aux légumes, ils font partie de tous les menus, et cela aussi bien en carême que hors carême, soit comme accompagnements de certains plats, soit comme plats à part, les plus fréquemment mentionnés étant l'artichaut, les fèves, le fenouil, l'asperge, l'épinard, le panais, en plus de divers types de laitue.

La liste des condiments utilisés pour la préparation des aliments jusqu'ici mentionnés comprend, outre l'huile, le vin, le verjus, le moût cuît, le vinaigre et la moutarde, diverses sortes d'“épices", du poivre, du sel noir, du sel blanc, du sucre, du safran, de la citronnelle, plusieurs variétés d'herbes dont le romarin et la sauge et, bien entendu, de l'ail et de l'oignon. Le lard, le saindoux, le beurre, les oeufs, la farine entrent dans la confection de nombreux plats, en particulier ceux relevant du pâtissier de la maison, et le riz semble utilisé surtout comme ingrédient de base de certains potages. Raffinement non négligeable, il est fait au moins une fois mention de fleurs et d'herbes servant à la décoration de la table cardinalice.

Il y avait sans doute à l'occasion des variantes apportées à ces menus - les jours de grande fête par exemple ou encore les jours où l'on recevait de grands 
personnages - une de ces variantes étant sans aucun doute la venaison qui ne figure sur aucun de nos menus, mais devait de temps à autre faire partie de ces derniers comme cela se faisait dans la plupart des grandes maisons de l'époque. ${ }^{30} \mathrm{~J}$ 'ai indiqué ailleurs ce qu'il en était chez le cardinal Bernardo Salviati. ${ }^{31}$ Combien plus riche de ce point de vue devait être la table d'un Alexandre Farnèse, lui qui était passionné de chasse et était entouré de proches qui partageaient cette passion et ne demandaient sans doute pas mieux que de le faire profiter de leurs plus belles prises. ${ }^{32}$

Nous ne possédons pas, comme chez Bernardo Salviati, la liste des vins consommés à la table du cardinal Farnèse. A un seul endroit il est spécifié que l'un des vins de sa cave est un "vin grec de qualité", sans doute l'un ou l'autre des meilleurs crus de la région de Naples, ${ }^{33}$ vin qui, en l'occurrence, est utilisé pour la préparation des tellines servies chaque midi durant la période quadragésimale. Mais il ne fait pas de doute que le cardinal Farnèse savait apprécier le bon vin et qu'il devait donc posséder une cave à l'avenant. En décembre 1575, découvrant au cours d'une chasse à la marina de Palo une botte de vin grec d'Ischia échouée par hasard sur la grève, il fait porter cette botte à Rome et, apprenant plus tard que le vin qu'elle contient est non seulement bien conservé, mais d'une qualité exceptionnelle, il baptise aussitôt cette botte du nom de "la Venturina" ("la petite fortune") et ordonne que son précieux contenu lui soit personnellement réservé. ${ }^{34}$ Caprice d'un moment, mais qui en dit long sur les goûts raffinés de ce grand personnage.

Ce que nous avons dit jusqu'ici de sa table montre bien que c'était d'ailleurs là une préoccupation quotidienne - car les menus décrits plus hauts, y compris ceux du carême, ne sont pas, comme on pourrait être tenté de le croire, des menus d'exception, mais bien le lot de tous les jours, en d'autres mots, ce qu'un habitué du palais de la Chancellerie se voyait servir midi et soir à longueur d'année. Nous avons comparé plus haut ce régime à celui qui existait au palais Salviati à la même époque. Celui du palais de la Chancellerie nous est apparu, en termes quantitatifs du moins, largement supérieur. Il semble bien, à la lumière de l'analyse que nous venons de faire des menus servis chaque jour dans cette même maison que cet avantage ait été en termes de qualité, tout aussi, sinon plus marqué..$^{35}$ Et sans doute faudrait-il aussi tenir compte du fait que le décor, l'ambiance, le service étaient nécessairement plus somptueux et raffinés au palais de la Chancellerie en raison des moyens plus importants, des personnels plus variés et plus nombreux et, bien entendu, de la qualité des lieux (palais, villas, jardins, "vignes") dont disposait le cardinal Farnèse.

Chose certaine, on pouvait difficilement trouver à Rome au milieu du XVI ${ }^{\mathrm{e}}$ siècle une cour que pratiquât avec plus de munificence, pour ne pas dire 
d'abandon, la vertu par excellence de toute bonne maison à l'époque: l'hospitalité. La cour pontificale, les cours de certains autres grands cardinaux: un Hippolyte et un Louis d'Este, un Ferdinand de Médicis, par exemple, se permettaient sans doute à l'occasion des extravagances de table que les chroniqueurs du temps s'empressaient de consigner, ${ }^{36}$ mais combien parmi elles assuraient un régime comparable à celui qu'on trouvait jour après jour, carême y compris, au palais de la Chancellerie? Ce n'est pas pour rien que les contemporains qualifaient Alexandre Farnèse de "grand cardinal". Il l'était à plus d'un titre mais il l'était surtout, comme nous venons de le voir, au chapitre de l'hospitalité.

D'autres titres de gloire sont souvent évoqués par les admirateurs d'Alexandre Farnèse, par exemple, sa grande générosité envers les pauvres et envers certains ordres religieux, en particulier les Jésuites, ${ }^{37}$ ou encore, ses talents de diplomate et son habileté politique au service des intérêts du Saint-Siège, mais également de sa propre maison. ${ }^{38} \mathrm{D}$ 'aucuns, à l'époque, préféraient insister sur les rapports privilégiés qu'il entretenait avec les humanistes et artistes de son temps dont il aimait ostensiblement s'entourer, qu'il n'hésitait d'ailleurs pas à combler de ses faveurs et à qui il se plaisait à passer des commandes, parfois même de très riches. On sait ce qu'il en fut d'écrivains tels que Paolo Giovio, Antonio della Mirandola, Annibale Caro, Onofrio Panvinio, d'architectes tels que Vignola ou della Porta, de peintres tels que Giorgio Vasari, Cecchino Salviati ou encore Taddeo et Federico Zuccari qui furent, les uns, membres de sa "famille", les autres, clients ou protégés, mais qui tous produisirent des oeuvres que nous pouvons encore aujourd'hui admirer, dont il fut sinon l'instigateur, du moins le principal soutien. ${ }^{39}$ Est-il besoin d'insister sur ce que pouvait représenter et représenta de fait pour la "famille" et les hôtes du cardinal la présence habituelle à ses côtés de ces hommes aux talents multiples et variés qui ne demandaient d'ailleurs qu'à le servir.

Vasari parle de soirées passées à Rome chez Alexandre Farnèse au cours desquelles il lui fut donné d'assister à des échanges du plus haut intérêt entre membres de la cour du cardinal et certains de ses hôtes sur l'art et la littérature et autres sujets susceptibles d'intéresser les beaux esprits du temps. ${ }^{40}$ Alexandre Farnèse et son entourage partageaient sans doute là-dessus les vue de Castiglione, l'auteur du Cortegiano, qui, on le sait, plaçait très haut dans la hiérarchie des arts de cour, celui de la conversation, ${ }^{41}$ art qu'on associait d'ailleurs de très près à l'époque aux plaisirs et raffinements de la table. ${ }^{42}$

Mais il y avait aussi la musique - Castiglione attachait de fait à cet art presque autant d'importance qu'à celui de la conversation ${ }^{43}-$ et, là encore, 
Alexandre Farnèse faisait figure de modèle, puisque sa famille comptait un nombre imposant de chanteurs et d'instrumentistes, plus d'une dizaine en 1544 , un peu moins peut-être en $1563,{ }^{44}$ auxquels il pouvait régulièrement faire appel pour son propre agrément ou celui de ses hôtes.

L'hospitalité ne se résumait donc pas chez lui aux simples plaisirs de la table, même si ces derniers représentaient, nous l'avons vu, le poste de loin le plus important du budget de sa maison. Elle incluait aussi diverses autres commodités de l'existence et puis surtout, comme nous l'avons souligné plus haut, des lieux et des décors somptueux conférant à ces plaisirs et à ces commodités un caractère peu commun, pour ne pas dire unique. En ce sens, la cour d'Alexandre Farnèse constituait bien pour la Rome de l'époque un modèle difficilement imitable, un modèle qui commençait d'ailleurs à être contesté - on n'a qu'à regarder ce qui se passait au même moment à la cour d'un Charles Borromée, par exemple, pour s'en rendre compte ${ }^{45}$-, mais ce modèle trouvait encore preneur et représentait encore pour plusieurs ce que pouvait, mieux ce que devait être la cour d'un prince, fût-il prince d'Église. Il y avait après tout une logique de la "grandeur" et Alexandre Farnèse montrait à sa façon jusqu'où cette logique pouvait aller quand on avait comme lui les moyens de la pousser jusqu'au bout. On comprend l'admiration et les critiques tout à la fois que son exemple ait pu susciter à l'époque, mais on comprend tout aussi bien la fascination qu'encore aujourd'hui nous éprouvons devant cet homme qui incarna pour ainsi dire la Renaissance à une époque ou la Renaissance, à Rome du moins, vivait son crépuscule. Mais, grâce à lui surtout, quel crépuscule!

\section{Université Saint-Paul}

\section{Note sur la consommation quotidienne par tête au palais de la Chancellerie}

Les moyennes de consommation par tête que nous avons établies plus haut à partir de notre document de base (AS, Filz. I: 81, filz. 32) sont bien évidemment des moyennes brutes qui demanderaient chacune à être modulées selon les individus et selon les catégories de personnes. Pour ce qui est du vin, notre base de calcul est la quantité totale de ce précieux liquide consommée ou, du moins, consignée chaque année au palais de la Chancellerie, soit, d'après les calculs de l'intendance du palais, environ $2000 \mathrm{hl}$ (fol. 10v). Si nous divisons ce total par les 270 individus inscrits au rôle de la cour du cardinal Farnèse, cela donne un peu plus de 21 par personne par jour.

Mais les choses ne sont pas aussi simples que cela. Tout d'abord, si nous examinons attentivement le rôle de la cour du cardinal, nous découvrons qu'il 
n'y a que 254 individus sur les 270 inscrits qui ont effectivement droit au "couvert". En revanche, il faut compter les invités, sans doute passablement nombreux chez le cardinal Farnèse qui, on le sait, avait une très large parentèle et clientèle. J'avais estimé à une dizaine en moyenne par jour le nombre de ces invités chez le cardinal Bernardo Salviati vers 1563-65. Cf. "La table d'un cardinal...”, p. 265 et note. Je crois que le chiffre de 15 serait plus vraisemblable dans le cas d'Alexandre Farnèse, ce qui nous ramènerait plus ou moins aux 270 "bouches" initiales et donc à la moyenne brute de 21 par personne par jour plus haut indiquée.

Mais il faut aussi tenir compte de quantités relativement importantes de vin utilisées pour la cuisine ou offertes en cadeaux - possiblement un cinquième du total comme chez le cardinal Salviati - ce qui obligerait à ramener la consommation brute par personne à environ 1,62 1. C'est le chiffre que je propose ici pour fin de comparaison avec le palais Salviati et Valladolid (cf. tableau C), sachant bien par ailleurs qu'il s'agit là d'un simple ordre de grandeur ne représentant sans doute qu'approximativement la réalité des choses.

Peut-être une autre estimation fournie, elle aussi, par les auteurs du budget de 1563, soit la "répartition" par tête de certaines denrées de base (pain, vin, huile) est-elle plus proche de cette réalité et faudrait-il la préférer à celle que je propose plus haut. En effet, la moyenne de 1,32 l suggérée par ce rôle nominatif paraît à première vue plus vraisemblable, mais cette moyenne ne tient visiblement pas compte de l" "extraordinaire" qui, au palais de la Chancellerie, équivalait, dans le cas du vin, à plus de $20 \%$ de la consommation totale. Tout bien considéré, il vaut peut-être mieux en rester à la moyenne de 1,62 l plus haut proposée, en se disant bien qu'il s'agit là d'une moyenne brute et d'une moyenne de "répartition" plutôt que de "consommation" au sens strict du terme. (Ces neuf litres de vin que se voient attribuer chaque jour les trois muletiers du cardinal sont-ils vraiment consommés par eux ou ne sont-ils pas plutôt partagés avec d'autres, des proches par exemple?). En se disant également que cette moyenne cache sans doute - nous l'avons constaté chez le cardinal Salviati et cela est tout aussi vrai chez le cardinal Farnèse - des écarts considérables entre individus et catégories d'individus, mais sans que nous puissions, dans le cas du cardinal Farnèse du moins, déterminer exactement ce que pouvaient être ces écarts. Chose certaine, le vin coulait plus généreusement et plus abondamment au palais de la Chancellerie qu'au palais Salviati.

Il semble qu'on se soit montré tout aussi généreux pour ce qui était du pain, mais, encore ici, il est difficile d'arriver à des chiffres tout à fait sûrs, puisque, comme dans le cas du vin, nous sommes confrontés à deux "estimations" 
divergentes, l'une fondée sur la quantité totale de pain achetée et distribuée chaque année par l'intendance du palais, soit environ $112000 \mathrm{kgs}$, ce qui équivaut à une consommation quotidienne brute par personne de 1136 grammes, l'autre sur le rôle nominatif plus haut mentionné qui attribue, lui, 707 grammes en moyenne par jour à chacun des individus inscrits à ce rôle. Cette dernière moyenne paraît trop basse pour les raisons que j'ai expliquées plus haut à propos du vin; l'autre est peut-être en peu trop élevée, mais elle paraît plus proche de la réalité et c'est finalement celle que j'ai choisi de retenir. (Une certaine quantité de pain servait sans doute aux aumônes, ne fût-ce que sous forme de "restes", mais quelle quantité au juste?)

Pour ce qui est de la consommation d'huile, notre estimation est fondée sur la seule information complète dont nous disposions, soit la quantité totale d'huile mise chaque année à la disposition des habitants du palais, soit en moyenne $4,26 \mathrm{cl}$ par personne par jour.

Les chiffres concernant la consommation de viande ou de poisson sont plus sujets à caution en raison du type de données dont nous disposions pour les établir. Nous nous en expliquons d'ailleurs plus haut (notes 23 et 24), mais nous croyons tout de même qu'ils représentent un ordre de grandeur tout à fait plausible et vraisemblable.

\section{Notes}

1. Pise. Archivio Salviati (= AS) Filz. I: 81 , fasc. 32 . Le document se présente sous la forme d'un fascicule grand format de 11 folios (recto-verso) subdivisé comme suit: 1) un rôle de la cour d'Alexandre Farnèse comportant la "répartition" quotidienne (ou hebdomadaire) par "bouche" de pain, de vin, d'huile (dans quelques cas seulement), de chandelle, le tout complété d'un companatico en argent pour les individus ayant des dépendants et de "rations" d'avoine et de fourrage pour ceux bénéficiant de "montures" (chevaux, mules, ânes) (fol. 1-3r); 2) le détail des menus quotidiens servis midi et soir à la table du cardinal (fol. $3 \mathrm{v}-4 \mathrm{r}$ ); 3) la liste détaillée des salaires, "provisions" et aumônes versés chaque mois par le cardinal (fol. 4v-5r); 4) la liste des pensions, cens et autres charges (ou dettes) du cardinal (fol. 54-7v); 5) la liste de ses créances (fol. $8 \mathrm{r}$ ); 6) ses entrées (ou revenus) (fol. $8 \mathrm{v}-10 \mathrm{r}$ ); 7) la liste des dépenses qui lui occasionnent l'entretien de sa cour et son entretien personnel (fol. 10v-11r). Riche document, mais qui n'est pas sans poser des problèmes de lecture et d'interprétation en raison de certaines particularités d'écriture et de vocabulaire, certaines lacunes également sur lesquelles nous aurons l'occasion de revenir plus loin. Il y a de fortes chances que le document en question ait été préparé par les services de comptabilité du cardinal, mais sous l'autorité du majordome du palais qui était, à l'époque, le premier responsable des questions d'intendance et de budget.

2. Mélanges de l'École française de Rome (Moyen Age, Temps modernes) (=MEFRM), 92 (1980) - I, p. 249-282.

3. Ibid., p. 276. 
4. Ibid., p. 264-265.

5. Ibid., p. 276.

6. Il nous manque une biographie d'Alexandre Farnèse, du moins digne de la stature de cet homme et du rôle qu'il fut appelé à jouer à Rome au XVI ${ }^{\mathrm{e}}$ siècle, Nous nous inspirons ici de l'excellent article de G. Alberigo, "Farnèse (Alessandro), dit Alexandre le Jeune", dans Dictionnaire d'histoire et de géographie ecclésiastique, XVI, Paris 1967, col. 608-615.

7. Sur ce palais, voir A. Schiavo, Il Palazzo della Cancelleria, Rome 1964. Plus récent et utile: C. Pericoli Ridolfini, Rione VI - Parione, II (Guide Rionali di Roma), Rome 1980 , p. 70-108.

8. Au sujet du long séjour d'Alexandre Farnèse au palais de la Chancellerie, voir L. von Pastor, Geschichte der Päpste, V. Fribourg-en-Brisgau, réimpr. 1956, p. 727, de même que C. Pietrangeli, Rione VII-Regola, II (Guide Rionali di Roma), Rome 1976, p. 62. Pour ce qui est du palais Farnèse comme tel, il faut préférer à l'ouvrage vieilli de $\mathrm{F}$. de Navenne (Rome, le palais Farnèse et les Farnèse, Paris, 1915), la monumentale histoire du palais publiée par l'École française de Rome à l'occasion de son centenaire: Le palais Farnèse, 3 tomes (en 5 vol.), Rome 1980-1982. Plus maniable, mais s'inspirant du précédent ouvrage: A. Puaux, Introduction au palais Farnèse, Rome 1983.

9. Voir C. Pietrangeli, op. cit., p. 58-62.

10. Notre calcul est fait à partir du rôle inclus dans le budget de 1563: AS Filz. I: 81, fasc. 32 , fol. 1-3v, 4v-5r. La répartition par catégories: gentilshommes et prélats, officiers et simples serviteurs est approximative, les indications fournies par le rôle n'étant pas toujours parfaitement claires. La même "famille" comptait en 1544277 membres, s'il faut en croire un rôle établi cette année-là par l'intendance du palais et qui se trouve aujourd'hui à la Bibliothèque du Vatican (=BAV): Barberini latini 5366, fol. 266v-267.

11. La cour du pape Pie IV comptait en 1652 plus de 1000 personnes, mais son neveu, le cardinal Borromée se contentait d'une "famille" d'environ 150 membres qu'il réduira d'ailleurs à une centaine en 1564. P. Partner, Renaissance Rome 1500-1599, Berkeley, Los Angeles, Londres 1976, p. 118, 135.

12. C'est le revenu comptabilisé dans le budget de 1563. Il s'agit surtout d'entrées bénéficiales telles, pour l'Italie, l'abbaye Tre Fontane de Rome (4 793, 50 écus) ou encore l'archevêché de Monreale en Sicile (20 900 écus), pour la France, l'archevêché d'Avignon (4 950 écus) ou l'abbaye de Grandselve (5 739 écus), mais il y a aussi des revenus de fonction, telles les entrées de la Chancellerie (6 800 écus) ou celles de la légation d'Avignon (4 950 écus), des pensions sur bénéfices, surtout portugais (7 340 écus au total), en plus de quelques revenus patrimoniaux, ceux du casale de Torre Vergata par exemple (800 écus. AS Filz. I: 81 , fasc. 32 , fol: $8 v-10$ r. Mais un examen attentif des créances du cardinal (43 407,48 écus au total) montre à l'évidence qu'il avait d'autres sources de revenus (prêts, rentes), donc des entrées réelles supérieures aux 78981 écus indiquées dans le tableau ci-dessus. Ibid., fol. 8v.

13. F. de Navenne, Rome, le palais Farnèse et les Farnèse, p. 250. 
14. J. Delumeau, Vie économique et sociale de Rome dans la seconde moitié du XVIe siècle, I, Paris 1957, p. 455.

15. C'est à-dire 70820 écus (entrées nettes) moins les charges diverses (pensions, cens, etc.) s'élevant à 8666 écus.

16. Ainsi le jeudi 7 juin 1657 Alexandre Farnèse y reçoit à dîner les cardinaux qui, la veille, avait participé au consistoire. BAV, Urbinati latini 1040, fol. 413 r.

17. Le cardinal y passe habituellement ses vacances d'été et y reçoit de nombreux visiteurs. A la fin de septembre 1569 , on y signale la présence de six cardinaux et du duc Ottavio Farnèse, frère du cardinal. Ibid., Urbinati latini 1041/I,fol. 155r. En 1578, il y reçoit le pape Grégoire XIII. J. Delumeau, op cit., p. 438.

18. En 1576, il offre une partie des joyaux estimés à 50000 écus présentés à la jeune épouse de Giacomo Boncompagni, fils du pape Grégoire XIII. BAV, Urbinati latini 1045, fol. 71v. En 1581, il verse 100000 écus comme "acompte" sur les 300000 promis pour la dot de sa nièce qui doit épouser la duc de Mantoue. J. Delumeau, op cit., p. 455.

19. Il comptera pendant un certain temps dans son personnel Alessandro Cesati, un des plus grands tailleurs de gemmes de l'époque. Cf. L. von Pastor, Geschichte der Päpste, V. p. 774. Selon Aldrovandi (1558), il possédait la plus riche collection d'antiquités de Rome. P. Pecchiai, Roma nel Cinquecento, Rome 1948, p. 413-414. Côté divertissements, il est souvent fait mention dans les documents d'époque de sa passion pour la chasse. En septembre 1578, à l'occasion de la visite de Grégoire XIII à Caprarola, il organise pour ce dernier plusieurs chasses, mais également des excursions de pêche et de régates sur le lac Vico. J. Delumeau, op. cit., p. 438.

20. J'ai expliqué ailleurs la distinction existant à l'époque entre "salaire" et "provision" et le cas particulier de certains "familiers" ou "proches" qui ne recevaient, eux, ni salaire ni provision, mais faisaient l'objet de "gratifications" diverses, en particulier sous forme de bénéfices ecclésiastiques, la plupart des "familiers" en question étant clercs, comme il se devait dans une cour cardinalice à l'époque. Voir à ce sujet ma communciation au colloque tenu à Québec en 1986 sur le thème: Travail et travailleurs au bas moyen âge ("Les métiers de cour à Rome à l'époque de la Renaissance", p. 25-28). La cour d'Alexandre Farnèse correspond en gros à ce modèle avec 90 'salaires'," une trentaine de "provisionés", une cinquantaine de "familiers" non salariés et non "provisionés", mais sans aucun doute généreusement "gratifiés", la rénumération des autres - une centaine au total - étant à la charge des individus compris dans ces trois catégories à titre de dépendants. En raison de cette "charge" et d'autres tout aussi onéreuses, la hiérarchie des salaires et des "provisions" inscrits au budget - de 9 à 76 écus par année dans le cas des salaires, de 24 à 396 dans le cas des "provisions" - ne doit pas être considérée comme reflétant très exactement le rang occupé à la cour par chacun des "salariés" ou des "provisionés". Giromalo Fioravanti, comptable de la maison, reçoit bien 76 écus par an comparativement aux palefreniers qui n'en reçcoivent que 12, mais il a deux dépendants et ils n'en ont aucun. Son salaire net est donc plutôt de l'ordre de 52 écus par an maximum comparativement aux 36 écus que reçoit $\mathbf{M}^{\circ}$ Giannetto, maître de l'écurie, ou aux 26 écus versés à Messer Pasquino, maître de la garderobe. Carlo Gualteruzzi, secrétaire du cardinal, a droit à une "provision" de 240 écus par an, mais il n'habite pas le palais de la Chancellerie et il a deux auxiliaires 
et sa propre famile à charge. A ce sujet voir O. Moroni, Carlo Gualteruzzi (1500-1577) e $i$ corrispondenti, Cité du Vatican 1984, p. 29. Il en va de même pour l'architecte Giacomo Vignola qui reçoit, lui, 192 écus par an. Il y a, en plus, certains cas "à part", difficiles à expliquer, tel cet "acquarolo" (ou acquiféraire) payé 106 écus par an, salaire à première vue exorbitant, mais peut-être s'agit-il là d'un individu n'habitant pas lui non plus le palais et ce "salaire" correspond-il en réalité à un contrat aux termes duquel le personnage en question s'engageait à fournir en eau les habitants et les divers services du palais.

21. A ce sujet, voir J. Delumeau, op cit., p. 443-444.

22. Voir plus loin: Note sur la consommation quotidienne par tête au palais de la Chancellerie, annexée au présent article.

23. Pour ce qui est de la viande, notre estimation est fondée, d'une part, sur le détail des menus quotidiens servis à la table du cardinal, d'autre part, sur le companatico en nature (dont 40 livres ou 13,56 kgs de viande par jour) offert aux autres membres de la famille, le tout calculé en fonction des 235 jours par année où l'on faisait gras, ce qui donne la moyenne brute par personne que nous proposons. Cette moyenne pourrait être trop basse, car, pour ce qui est de la table du cardinal, soit 90 personnes au total, il faudrait de toute évidence ajouter la venaison qui ne figure pas sur les menus types retenus pour fins de calcul par l'intendance du palais, mais qui devrait sans doute assez souvent y trouver place, et pour ce qui est des autres membres de la "famille", ils pouvaient très bien utiliser le companatico en argent qui leur était également versé $(4,36$ baiocchi par jour) pour compléter la maigre ration de viande qui leur était consentie.

24. Calcul fondé sur les menus types indiqués par l'intendance du palais pour le temps du carême, mais qui valaient sans doute aussi pour les autres jours où l'on faisait maigre, soit 130 jours au total.

25. Pour ce qui est des données relatives au palais Salviati et à la ville de Valladolid en Espagne, voir notre article plus haut cité: "La table d'un cardinal...", p. 277 et notes.

26. En effet, dans la société fortement hiérarchisée de l'époque, seuls des personnages d'un certain rang pouvaient prétendre partager la table d'un prince de l'Église.

27. Pour les renseignements qui suivent, voir AS Filz. I: 81 , fasc, 32 , fol. $3 \mathrm{v}-4 \mathrm{r}$.

28. "Spicola" dans l'original. On mange encore aujourd'hui en Italie ce poisson qui est de la famille du loup de mer.

29. Pour "marangone".

30. A ce sujet, voir P. Tucoo-Chala, "La chasses au moyen âge" dans $L^{\prime}$ histoire, $\mathrm{n}^{\circ} 28$, nov. 1980 , p. 33-38.

31. "La table d'un cardinal...", p. 270-271.

32. Voir note 19. Comme indice de l'importance que le cardinal accordait à la chasse, on trouve dans son personnel en 1563, un fauconnier, des bracchieri (piqueurs), des canattieri (valets de chiens) en plus d'un capocaccia (maître de chasse). AS Filz. I: 81, fasc. 32 , fol. $2 v$. 
33. Ibid., fol. 4r. Les vins "grecs" provenaient en effet de la région de Naples et étaient hautement considérés à l'époque ... Cf. J. Delumeau, Vie économique et sociale... I, p. 117.

34. BAV, Urbinati latini 1044, fol. 703v (Rome, 21 déc. 1575).

35. Mais - la question mérite d'être posée - de quelle valeur nutritive était ce régime somptueux, ce régime de "riche"? J'avais déjà noté, à propos du cardinal Bernardo Salviati, à quel point une consommation trop abondante de vin et de viande était probablement la cause des ennuis de santé qu'il éprouva au cours des dernières années de sa vie (goutte, troubles d'ordre vasculaire) et qui furent probablement la cause de sa mort en 1568. Voir "La table d'un cardinal...", p. 264 et 280. La consommation de vin et de viande semble avoir été encore plus abondante chez le cardinal Farnèse. Or ce dernier ne mourra qu'en 1589 à l'âge respectable de 69 ans. Mais peut-être Alexandre Farnèse jouissait-il d'une meilleure constitution ou s'était-il lui-même contenté d'un régime plus frugal que celui de son collègue Salviati ou que celui qu'il tenait à offrir à ses hôtes. Il n'en reste pas moins que sa "table" comme celle de la plupart des "grands" et des riches de l'époque ne répondait pas aux normes de ce que nous considérerions aujourd'hui comme un régime équilibré. Trop riche en calories, en protéines, en lipides, en glucides et en alcool et habituellement pauvre en vitmaines et en minéraux, la cuisine servie dans ces grandes maisons était à la source de nombreuses maladies qui sévissaient dans les milieux aristocratiques et de haute bourgeoisie du temps, entre autres, la goutte, divers types de rhumatismes, les lithiases rhénales ou hépatiques en plus de troubles cardio-vasculaires de toutes sortes. A ce propos, voir l'excellente étude de L. Balletto, Medici e farmaci, scongiure ed incantesimi, dieta e gastronomia nel medioevo genovese, Gênes 1986, p. 179-246 (mais, plus particulièrement, p. 216-218). Cet "envers du décor" auquel nous sommes sans doute aujourd'hui plus sensibles que ne pouvaient l'être Alexandre Farnèse et ses contemporains doit aussi être considéré quand on s'intéresse au régime de vie de ses derniers.

36. A ce sujet, voir J. Delumeau, op cit., p. 439-442. Sur les deux cardinaux d'Este, Hippolyte II (1509-1572) et Louis (1538-1586), seigneurs fastueux de Rome et surtout constructeurs de l'imcomparable villa d'Este à Tivoli, voir V. Pacifici, Villa d'Este, Tivoli 1923, Ippolito II d'Este, Cardinale di Ferrara, Ibid., [s.d], et Luigi d'Este, Ibid., 1952. Sur Ferdinand de Médicis, futur grand-duc de Toscane, voir le témoignage d'un contemporain bien informé, P. Usimbardi, "Istoria del Granduca Ferdinando I", dnas Archivio Storico italiano, VI (1880), série IV, p. 365-401.

37. Cf. BAV, Urbinati latini 1057, fol. 120r (Rome, 4 mars 1589). Sur la participation du cardinal à la construction du Gesù à Rome, voir P. Pecchiai, Il Gesù di Roma, Rome 1952. Sur le sens à donner à la protection accordée aux Jésuites, cf. G Alberigo, art. cit., col. 613-614.

38. Ibid., col. 610-613.

39. Dans ses Due Dialoghi publiés à Camerino en 1564 et dédiés à Alexandre Farnèse, Giovanni Andrea Gilio n'hésite pas à qualifier le cardinal de plus grand protecteur des humanistes de son temps et de vanter son affabilité, bénignité et générosité sans pareille (fol. 7v). Les écrivains Giovio, della Mirandola, Caro, Panvinio, pour ne citer que ceux-là furent de la cour du cardinal et Caro, en partiulier, y remplit longtemps l'office de secrétaire. Une partie de sa correspondance a été publiée: Lettere familiari, 3 vol., 
p.p. A. Greco, Florence 1957-1961. Il en est de même de celle d'un autre secrétaire, Carlo Gualteruzzi, mentionné tout comme Panvinio d'ailleurs dans le rôle de cour faisant partie du budget de 1563. Cf. O. Moroni, Carlo Gualteruzzi (1500-1577) e $i$ corrispondenti, Cité du Vatican, 1984. Ces deux correspondances permettent de mesurer l'étendue du réseau d'artistes et d'humanistes “protégés" par le carinal Farnèse. Sur commande de ce dernier, Vasari et Salviati travaillèrent à la décoration du palais de la Chancellerie, Federico Zuccari, à celle de l'Église voisine de San Lorenzo in Damaso. C. Pericoli Ridolfini, Rione VI-Patrione, II, p. 96-102, 112. A la demande du même ou de sa famille, Giacomo della Porta travailla comme architecte à la conception et à la réalisation de l'oratoire du Crocifisso, du Gesù, du palais Farnèse et de l'église Santa Maria Scala Coeli. V. Tiberia, Giacomo della Porta. Un architetto tra manierismo e barocco, Rome 1974, p. 25, 29, 33, 37, 40. Vignola et les frères Zuccari se signalèrent surtout, le premier, comme architecte, les seconds, comme décorateurs, de l'incomparable villa de Caprarola, si chère au coeur d'Alexandre Farnèse. A noter que Caro et Panvinio de même que Fulvio Orsini, autre humaniste protégé par le cardinal, fournirent les principaux thèmes iconographiques utilisés pour la décoration de la villa. J. Recupero, Il Palazzo Farnese di Caprarola, 1975, passim.

40. P. Pecchiai, Roma nel Cinquecento, p. 324.

41. Le Libro del Cortigiano se présente d'ailleurs comme une longue conversation et donc comme une sorte de démonstration de cet art par excellence de la vie de cour. Il servira de modèle à d'autres ouvrages du génre aux $\mathrm{XVI}^{\mathrm{e}}$ et $\mathrm{XVII}^{\mathrm{e}}$ siècles qui, eux aussi, insisteront sur l'importance de cet art. A ce sujet, voir A. Quondam, "La forma del vivere", dans La Corte e il "Cortegiano", II, Rome 980 p. 15 suiv.

42. A ce sujet, voir J.F. Solnon, La cour de France, Paris 1987, p. 66, 92-94, 104-106.

43. Cf. E.E. Lowinsky, "Music in Renaissance Culture", dans Renaissance Essays, New York. 1968, p. 344.

44. Des dix musiciens mentionnés dans le rôle de 1544 , deux sont identifiés comme instrumentistes ("chontrabasso", "suonator di leuto"). BAV, Barb. lat. 5366, fol. 266v. Un seul courtisan est identifié comme musicien dans le rôle de 1563, mais il y en avait certainement d'autres qui, malheureusement, cette fois ne sont pas identifiés comme tels. AS, Filz. I, 81, fasc. 32, fol. 1-3v.

45. A partir de 1563, Charles Borromée réduisait considérablement son train de vie et les dimensions de sa cour. Il finit par convaincre son oncle, Pie IV, d'en faire autant. Ce nouveau modèle parut à plusieurs à l'époque plus conforme au nouvel idéal clérical proposé par le concile de Trent. Cf. R. Mols, "Charles Borromée (Saint)", dans Dictionnaire d'histoire et de géographie ecclésiastique, XII (1953), col. 494-495. 